\title{
An Empirical Evaluation of Touch and Tangible Interfaces for Tabletop Displays
}

\author{
Aurélien Lucchi, Patrick Jermann, Guillaume Zufferey, Pierre Dillenbourg \\ CRAFT - EPFL \\ aurelien.lucchi, patrick.jermann, guillaume.zufferey, pierre.dillenbourg @epfl.ch
}

\begin{abstract}
Tabletop systems have become quite popular in recent years, during which there was considerable enthusiasm for the development of new interfaces. In this paper, we establish a comparison between touch and tangible interfaces. We set up an experiment involving several actions like translation and rotation. We recruited 40 participants to take part in a user study and we present our results with a discussion on the design of touch and tangible interfaces. Our contribution is an empirical study showing that overall, the tangible interface is much faster but under certain conditions, the touch interface could gain the upper hand.
\end{abstract}

\section{Author Keywords}

$\mathrm{HCI}$, touch, tangible, interface

\section{ACM Classification Keywords}

H.5.2 Information Interfaces and Presentation: Miscellaneous

\section{INTRODUCTION}

The quest for an ideal interface between humans and computers has garnered a lot of attention. Many studies ([3],[9]) have been conducted to assess the performance of interfaces (mouse, pen, tablet, touch, tangible). To our surprise, hardly any performance studies about tangible interfaces exist, in spite of their many provable learning benefits [13]. Another argument in favor of tangible interfaces is the evidence showing they can take advantage of a broader range of human abilities than other interfaces. In this paper, we will try to answer the following questions. Is there a statistical and substantial improvement in tangible interfaces relative to other ones; and how generalizable is this effect ? This led us to conduct a study comparing speed and error rates for two manipulation devices, namely a touch interface and a tangible interface.

Most of the previous studies comparing the performance of the mouse and other devices based their evaluation on Fitt's law [5]. Fitt's law defines the time to acquire a target as a function of the distance traveled (between the starting position and final target position) divided by the target size. We believe that tangible interactions are too complex to be measured by Fitt's law. Therefore, we replaced the measurement of distance by measurements of a certain number of predefined actions such as translation and rotation.

We start by reviewing the existing work related to the comparison of different input interfaces, followed by a description of our system, the study and the results. Finally, we will discuss the implications of our work and propose some guidelines for the design of tangible interfaces.
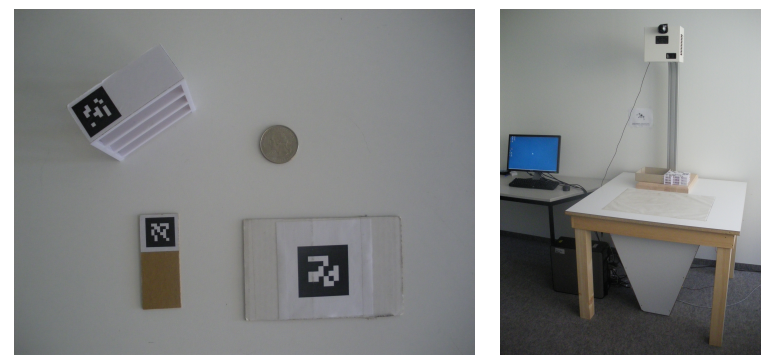

(a) The tangible objects used for our experi- (b) Picture of the table built ments with a US quarter for scale (top-left: a for our experiment shelf, bottom-left: a wall, bottom-right: submit card).

Figure 1. Our Experimental Setup

\section{RELATED WORK}

The Digital Desk [24] was the pioneering work to integrate physical and digital documents on a table. The authors brought some of the functionalities we typically associate with graphical user interfaces (GUIs) onto the physical desktop. The concept of a graspable or tangible user interface (TUI) was later introduced by Fitzmaurice et al to develop the largely influential Bricks system [7]. Other systems relying on this concept were later developed (BUILD-IT [17], Urp [23],...). All those interfaces take advantage of human's early ability to interact with physical objects.

Touch screen interfaces have become quite popular during the last few years. The reasons for this success are probably related to the robustness and the longevity of the interface. However, touch screen interfaces have the reputation of being error prone. The fat finger problem has been designated as largely responsible for this issue and many papers have addressed this ([25], [1]). Previous studies showed that 
the touch screen interface was among the fastest interfaces but also the least accurate unless special design strategies were conceived ([19], [14]). The first formal study of tangible interfaces was done by Fitzmaurice and Buxton [6] who compared a regular mouse-operated GUI application and a graspable user interface in a fixed-duration target-tracking task. They concluded that the mouse is a general all-purpose device. Tangible interfaces are regarded as more specific devices that can be used to perform a task very well but are only suited for limited learning domains such as programming, narrative, molecular biology/chemistry and dynamic systems [13]. One of the real challenges for tangible interfaces is to understand for which tasks and domains they are relevant.

Very little interest has been shown in studying the performance of tangible interfaces. Terrenghi et al [22] opened up the field by exploring the benefits of physical interfaces on interactive surfaces. They compared a digital and a physical interface for a puzzle and a photo sorting task and they found that the physical interface was significantly faster for sorting pictures but slightly slower when completing a puzzle. They hypothesized that this was because of the difficulty of manipulating the smaller pieces. They stressed the importance of bimanual interactions and suggested that special design rules had to be used in order to benefit from this interaction with the touch interface.

\section{THE MULTI-TOUCH TANGIBLE TABLE}

In order to compare tangible and touch interfaces, we built a top-projection tabletop system supporting both interfaces. The table provides a work surface of $49.6 \mathrm{~cm} \times 37 \mathrm{~cm}$. The system uses a camera mounted on top of the table to detect tagged objects placed on the table using ARTag, a fiducial marker tracking library [4]. The table is also an interactive surface as it can track multiple fingers using FTIR technology [10]. A real-time fingertip tracking method had to be implemented to allow for a natural interaction and to avoid user frustration. A second camera sensitive to infrared light was placed below the table. The table consists of a sheet of Plexiglas and infrared LEDs shining into the sides of the Plexiglas. When a finger comes into contact with the surface, the light ray is reflected at the camera, creating a blob in the captured image (see figure 2). A background subtraction algorithm using a mixture of Gaussians [21] is then used to detect these blobs.

\section{Methodology}

In order to establish a fair comparison, the software developed for both interfaces had to be as identical as possible. To this end, we used physical metaphors for the actions used for the touch interface and presented in table 1. Two different types of objects were used for the experiment. The first type of object is a shelf (such as the one used in warehouse for the storage of goods) and the second type of object is a wall. Further, we assume that virtual shelves are non-rescalable objects whereas virtual walls can be rescaled.

Figure 3 shows the screen of the application developed for the touch interface. A toolbar contains some items that can

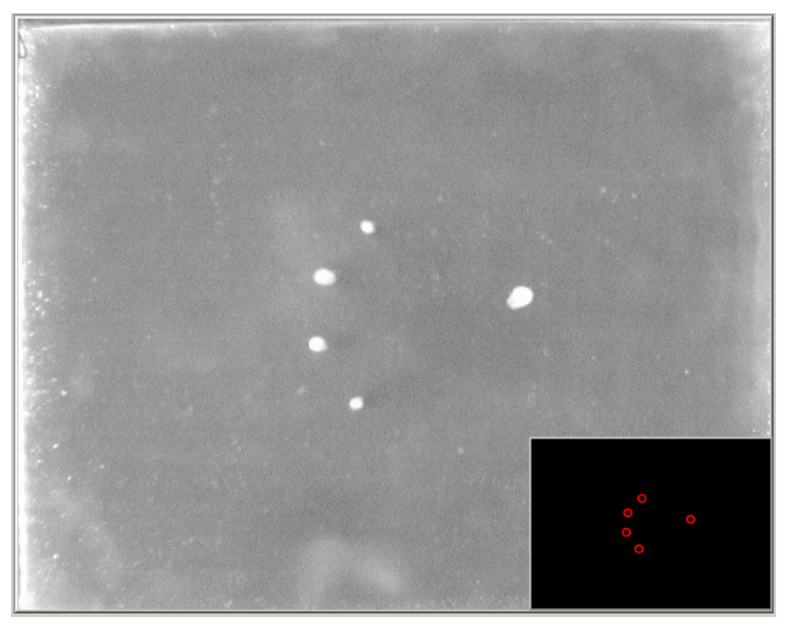

Figure 2. Image captured by the infrared camera and the blob detection in the lower-left corner.

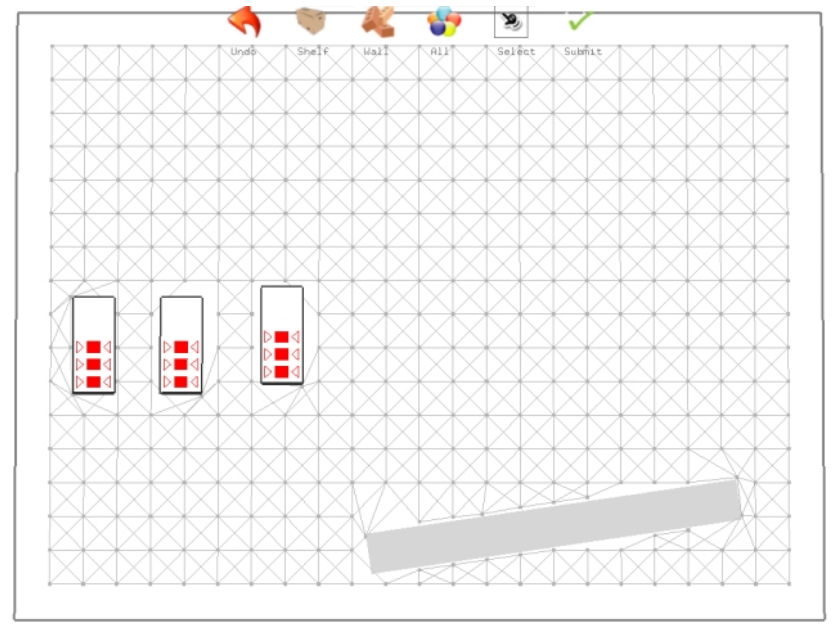

Figure 3. Screenshot of the simulation tool with three shelves and one wall. The toolbar at the top contains icons annotated with a short text.

be dragged and dropped in the work area to create new objects. It should also be noticed that a grid was drawn in the background to help users place objects accurately. We also implemented a snapping option that forced the position of an object to be rounded to the nearest grid point. During the testing phase, we realized that simple things (such as maximizing the space between items or displaying an explicative note below each item) could greatly improve the ease of interaction.

Two conditions differed in the tangible interfaces :

- The toolbar was removed and the icons used to create objects were replaced by tangible objects.

- Unlike the virtual walls, the tangible walls were of course non-scalable.

As shown in figure 1(a), the tangible shelves are small-scale versions $(66 \mathrm{~cm} \times 57 \mathrm{~cm} \times 24 \mathrm{~cm})$ of the real ones. The virtual walls were made of a thin piece of cardboard $(66 \mathrm{~cm} \times 57 \mathrm{~cm} \times 2 \mathrm{~cm})$. 
Compared to the tangible shelves, they could be described as planar objects. In order to give a feedback to the user, we augmented the scene by drawing on the tagged objects.

Table 1. Description of the gestures used for both interfaces

\begin{tabular}{|c|c|c|}
\hline Action & Touch & Tangible \\
\hline Addition & $\begin{array}{l}\text { Simply place a finger on } \\
\text { one of the items in the } \\
\text { toolbar and then drag the } \\
\text { object to the desired posi- } \\
\text { tion. }\end{array}$ & $\begin{array}{l}\text { Grab a tangible } \\
\text { object placed at } \\
\text { the top of the ta- } \\
\text { ble and place it } \\
\text { in the work area. }\end{array}$ \\
\hline $\begin{array}{l}\text { Lasso selec- } \\
\text { tion }\end{array}$ & $\begin{array}{l}\text { A lasso selection can be } \\
\text { started by keeping a finger } \\
\text { pressed for 500ms. The } \\
\text { surrounding objects will } \\
\text { then be selected after re- } \\
\text { leasing the finger. A line } \\
\text { was drawn as visual feed- } \\
\text { back while selecting. }\end{array}$ & Not applicable. \\
\hline Translation & $\begin{array}{l}\text { Select the object with one } \\
\text { finger and move the finger } \\
\text { to the desired location. }\end{array}$ & Natural gesture. \\
\hline Rotation & $\begin{array}{l}\text { Place fingertips at both } \\
\text { ends of the object and per- } \\
\text { form a rotation move. }\end{array}$ & Natural gesture. \\
\hline Scaling & $\begin{array}{l}\text { Place fingertips at both } \\
\text { ends of the object and } \\
\text { move them apart. }\end{array}$ & $\begin{array}{l}\text { Place several ob- } \\
\text { jects next to each } \\
\text { other. }\end{array}$ \\
\hline Removal & $\begin{array}{l}\text { Simply drag and drop the } \\
\text { object outside the screen } \\
\text { area. }\end{array}$ & $\begin{array}{l}\text { Remove the } \\
\text { object from the } \\
\text { work area. }\end{array}$ \\
\hline $\begin{array}{l}\text { Undo previ- } \\
\text { ous action }\end{array}$ & $\begin{array}{l}\text { A button is available in the } \\
\text { toolbar. }\end{array}$ & Not applicable. \\
\hline Submit & $\begin{array}{l}\text { A button is available in the } \\
\text { toolbar. }\end{array}$ & $\begin{array}{l}\text { Show a tagged } \\
\text { card to the cam- } \\
\text { era. }\end{array}$ \\
\hline Select All & $\begin{array}{l}\text { A button is available in the } \\
\text { toolbar. This option al- } \\
\text { lows users to select all the } \\
\text { virtual objects. }\end{array}$ & Not applicable. \\
\hline Adjustment & \multicolumn{2}{|c|}{$\begin{array}{l}\text { A small translation of a few centimeters } \\
\text { to bring two objects in contact. }\end{array}$} \\
\hline
\end{tabular}

\section{EXPERIMENT}

We asked 40 students (14 females and 26 males) from the university campus to participate in the experiment for which they each received 20 Swiss Francs. The students had different backgrounds (technical and non-technical) and they were aged from 14 to 30.15 of them reported owning a touchscreen device and 32 reported having used a touch interface before the experiment.

The subjects had to implement a series of 40 layouts shown in figure 5. Even though the same experiment was performed for the two interfaces, the introductory part for the touch interface included an additional four minutes video to explain the different actions available. The subjects were told that

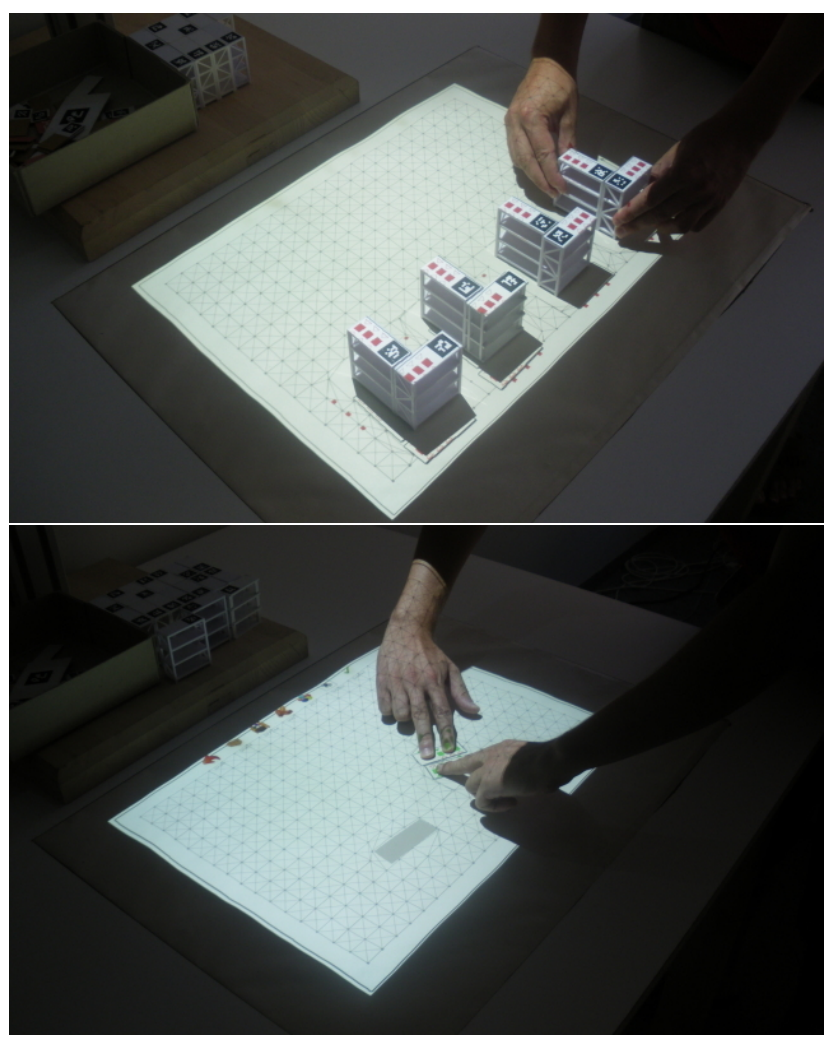

Figure 4. Pictures of a participant using the tangible interface (top) and the touch interface (bottom).

the goal of the study was to measure both speed and accuracy. After a short familiarization session (two minutes), they started the experiment with an empty shop floor. Once they had laid out the first warehouse, they had to submit their design and move to the next one. After submitting a design, the screen was flashed for one second to report to the user that his action had been recorded. The subjects were told to complete the experiment as quickly and as accurately as possible, but no time limit was given. The experiments were video-taped and log files recorded the actions performed by the user with the completion time. The tasks were designed so that we could easily compare the time taken between the different layouts. To this end, the number of moves from one layout to the next one was restricted to $1,4,8$ and 16 moves. Several types of action presented in table 1 were considered for the design of the layouts. Two additional combinations of action; a rotation plus a translation and an addition plus a rotation were also included.

All the participants had to perform the experiment with the two interfaces. We balanced the order of the two experiments, and our analysis of the results showed that the order did not significantly impact performances. This led us to ignore this effect in the following analysis.

\section{RESULTS}

The results presented in this section are based on a statistical analysis of the $\log$ files recorded during the experiments. A few comments given by the participants are also reported. 


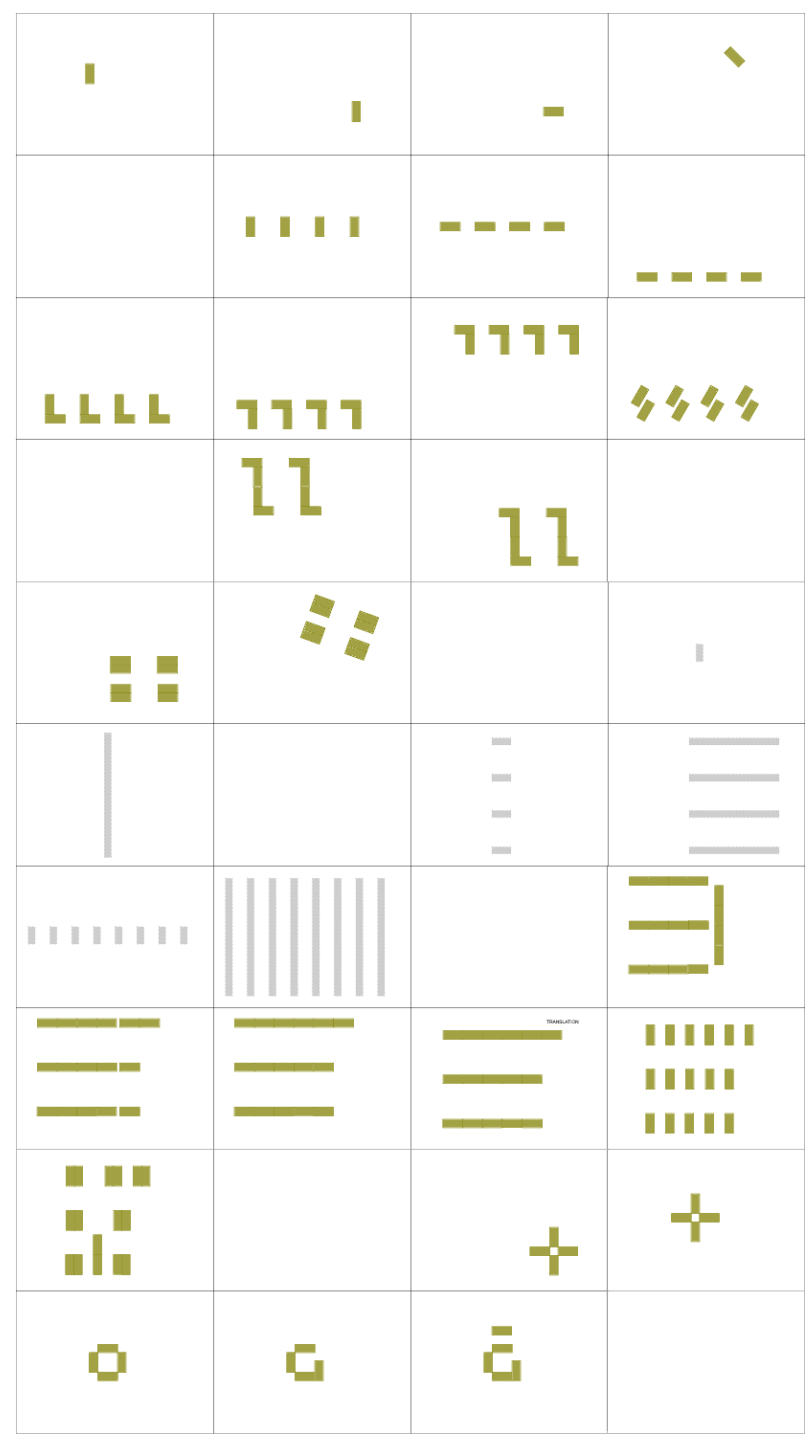

Figure 5. Series of layouts given to the participant. The grey and brown colors represent, respectively, the walls and the shelves. The grid present during the experiment was removed in order to improve the clarity of the picture.

The dependent variables are :

- Completion time for the overall experiment

- Completion time for each action.

- Accuracy (degree to which the reproduction reflected the model).

\section{Analysis of the completion time for the overall experi- ment}

All the participants were faster with the tangible interface but it should be noted that six of them finished the touch experiment within 2 minutes of the completion time of the tangible experiment. The best completion time for the touch interface was 18.50 minutes while the best time for the tangible interface was 13.40 minutes (see further details in table 2). The analysis of the video revealed that the fastest participants were very comfortable with the touch interface, making heavy use of the different options available (lasso selection, "undo" and "select all" buttons).

Table 2. Completion time (in minutes) for the whole experiment for touch and tangible interfaces

\begin{tabular}{|c|c|c|c|c|}
\hline & Min. & Median & Mean & Max. \\
\hline Tangible & 13.41 & 24.72 & 23.82 & 38.34 \\
\hline Touch & 18.50 & 29.63 & 28.74 & 42.97 \\
\hline
\end{tabular}

Figure 6 shows detailed plots of the average completion time per layout for shelves and walls. We can conclude that manipulating the tangible shelves (average time: 19.8 seconds) was much easier compared to the tangible walls (average time: 37.0 seconds). Furthermore, rescaling virtual walls proved to be faster than placing several tangible walls next to each other. We also observed a greater variance in the completion time for the virtual objects. This proves that some participants had more practice with touch interfaces or had better learning abilities than the others. In contrast, the more uniform distribution for the tangible interface shows that fewer disparities exist among participants.
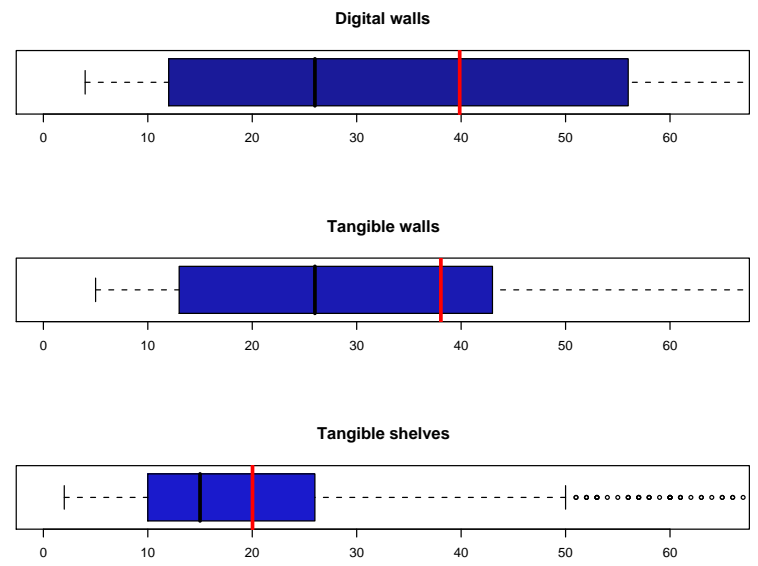

Digital shelves

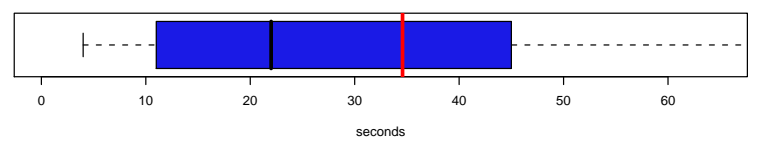

Figure 6. Box plots showing the accuracy for touch and tangible by differentiating walls and shelves. The red line shows the mean

For the translation action, we plotted the time for participants to complete the actions against the number of actions to be performed in figure 8 . Notice that the time taken grows linearly until the number of actions equals 8 . Surprisingly, the completion time for 16 objects is almost identical to the completion time for 8 objects. After analyzing the videos, we concluded that this was primarily due to the use of the "select all" button or the "lasso selection". Even participants who were not very keen to use those options in the past made an exception in this particular case. 


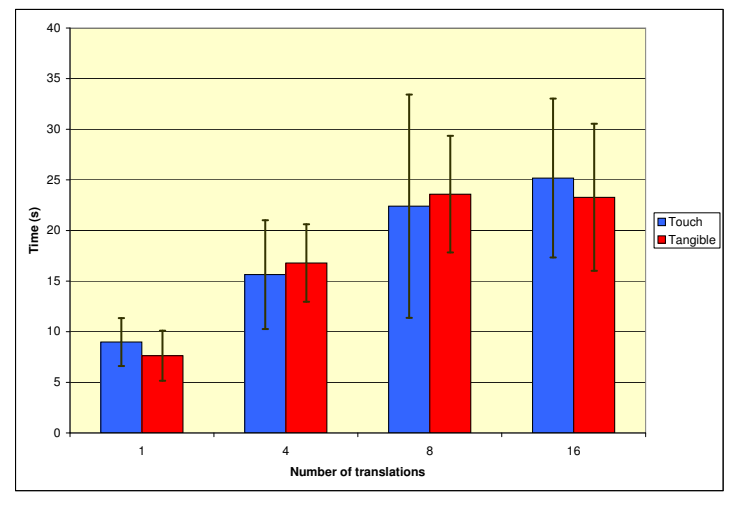

Figure 7. Bar graphs of movement time for the touch and tangible interfaces. Error bars represent standard deviations of the mean.

\section{Analysis of the completion time for individual actions}

In this section, we will study the time difference between the touch and tangible interface to complete the overall experiment. The dependent variable denoted $y$ is defined as $y=$ Time_tangible - Time_touch. Due to the nature of our experiment, which relies on repeated measures, we tried to fit a linear mixed-effects model [20] to our data. This type of multiple linear regression attempts to model the relationship between the dependent variable $y$ and the explanatory variables (number of actions).

$$
y=b_{0}+\sum_{i=1}^{n} b_{i} X_{i}
$$

Equation 1 will also be denoted as :

$$
y \sim \sum_{i=1}^{n} b_{i} X_{i}
$$

The variables $X_{i}$ represent the number of each action measured in the experiment. The intercept $b_{0}$ estimates the value of $y$ when all the predictors are 0 . The regression coefficients $b_{i}$ evaluate the sensitivity of $y$ to change in $X_{i}$.

Table 3 shows the correlation between y and the predictors for the layouts using walls as input objects. We can conclude that for each additional rotation, the time difference between tangible and touch increases by 2.67 seconds in favor of the touch interface. Our hypothesis is that as the number of actions increase, people are more prone to use special gestures. As no special gestures were available in our touch interface to add objects, this explains why the time difference increases by 8.15 seconds per addition in favor of the tangible interface.

As illustrated in table 4, the same conclusions can be drawn for the shelves. Indeed, increasing the number of translations and deletions privileges the touch interface. Con-
Table 3. $y_{\text {walls }} \sim$ Rotate + Add + Remove. All measurements are in seconds.

\begin{tabular}{|c|c|c|c|c|}
\hline & Value & Std.Error & t-value & p-value \\
\hline (Intercept) & 9.65 & 2.85 & 3.378 & 0.0008 \\
\hline Rotate & $\mathbf{2 . 6 7}$ & 1.73 & 1.543 & 0.1239 \\
\hline Add & $\mathbf{- 8 . 1 5}$ & 0.88 & -9.206 & 0.0000 \\
\hline Remove & -0.13 & 0.85 & -0.147 & 0.8826 \\
\hline
\end{tabular}

versely, adding objects favors the tangible interface. Another hypothesis to explain this observation is that it is relatively easy to grab several tangible objects (shelves or walls) in one hand compared to the virtual objects which have to be selected sequentially one by one.

Table 4. $y_{\text {shelves }} \sim$ Rotate + Translate + Rotate:Translate + Add + Add:Rotate + Remove + Adjust. (X:Y) denotes a combination between the 2 actions $X$ and $Y$. All measurements are in seconds.

\begin{tabular}{|c|c|c|c|c|}
\hline & Value & Std.Error & t-value & p-value \\
\hline (Intercept) & -5.49 & 1.53 & -3.573 & 0.0004 \\
\hline Rotate & -0.99 & 0.23 & -4.356 & 0.0000 \\
\hline Translate & 0.47 & 0.21 & 2.175 & 0.0298 \\
\hline Add & -4.80 & 0.25 & -18.573 & 0.0000 \\
\hline Remove & 0.27 & 0.21 & 1.257 & 0.2087 \\
\hline Adjust & -1.95 & 0.45 & -4.277 & 0.0000 \\
\hline Rotate:Translate & -0.61 & 0.06 & -9.037 & 0.0000 \\
\hline Rotate:Add & 0.14 & 0.07 & 2.068 & 0.0388 \\
\hline
\end{tabular}

\section{Analysis of the accuracy}

The similarity between the original layouts given to the participants and the reproduction was measured using the sum of squared differences (SSD) and then normalized to obtain a percentage. The error is defined as error $=100 \%-$ similarity. The dependent variable denoted $y$ is defined as $y=$ Tangible_Error - Touch_Error.

Table 5. $y \sim$ Rotate + Translate + Rotate:Translate + Add + Add:Rotate + Remove + Adjust. All measurements are in seconds.

\begin{tabular}{|c|c|c|c|c|}
\hline & Value & Std.Error & t-value & p-value \\
\hline (Intercept) & -2.42 & 0.93 & -2.608 & 0.0096 \\
\hline Rotate & 0.20 & 0.13 & 1.532 & 0.1268 \\
\hline Translate & 0.28 & 0.12 & 2.353 & 0.0193 \\
\hline Add & 0.25 & 0.12 & 2.144 & 0.0329 \\
\hline Remove & -0.07 & 0.53 & -0.138 & 0.8901 \\
\hline Adjust & 0.59 & 0.24 & 2.38 & 0.0177 \\
\hline Rotate:Translate & -0.01 & 0.03 & -0.334 & 0.7380 \\
\hline Rotate:Add & -0.02 & 0.03 & 0.578 & 0.5632 \\
\hline
\end{tabular}

Figure 8 shows that the touch was slightly less accurate but a more significant observation can be made. Participants were more accurate when using the shelves with both interfaces. We also noticed that the biggest errors were made with the digital walls that were re-scalable. We can not fully explain this effect though it could be ascribed to the software. 
We performed the same type of multiple linear regression analysis as described for the time analysis but we did not find any significant difference (the results are given in table 5). This supports the idea that the fat finger problem only applies to interfaces designed for small touch screens.
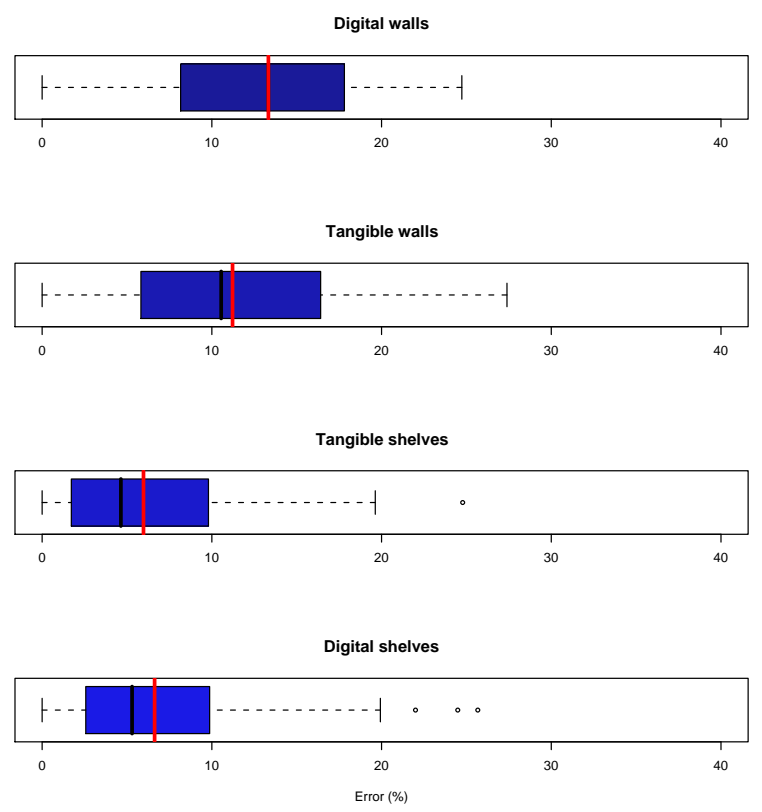

Figure 8. Box plots showing the errors for touch and tangible by differentiating walls and shelves. The red line shows the mean and error bars represent standard deviations of the mean.

\section{User preferences}

At the end of each experiment, we asked participants to give their opinion about the two interfaces. Two series of questions were given.

For the first series of questions, participants ranked each factor on a Likert scale. All the participants agreed that the tangible interface was easy to use but the answers were more varied for the touch interface. This result supports the idea that tangible interfaces are easier to learn compared to touch interfaces. Overall, participants reported to have more fun with the touch interface, which could be explained by the fact that the touch interface was more challenging to learn compared to the tangible interface whose use was obvious to most of the participants. However, some participants reported that both interfaces could become irritating after some time. When asked for more details about their answers, they said that both interfaces could cause some fatigue.

A second series of questions asked the participants to provide their subjective judgment on speed, ease of the tasks and fatigue caused by the application. Two observations can be made. First, participants reported that they were more successful with the tangible interface but no significant difference was found in the statistical analysis. More participants admitted to being more stressed and irritated with the touch interface. This could be explained by the lack of fa- miliarity with touch interfaces.

\section{DISCUSSIONS}

In this section, we will discuss possible causes and implications of our findings as summarized in table 6 .

Table 6. Qualitative comparison between tangible and touch interfaces

\begin{tabular}{|ll|}
\hline Touch & Tangible \\
\hline \hline Generalize better to a wide & Specific purpose of applica- \\
variety of application & tions \\
Easier to design & Good design is challenging \\
& Generally more efficient and \\
& faster to use \\
Less intuitive because of & Actions are more sponta- \\
lack of familiarity (which & neous according to our past \\
necessitates training) & experience \\
Non persistence & Persistence (can outlive the \\
& execution of the program) \\
Visual feedback & Visual, tactile and audio \\
& feedback \\
\hline
\end{tabular}

Special purposeness of tangible objects make them difficult to re-use for a different application and that probably explains why so many tailored tangible applications have been proposed ([16], [2], [12], [15]).

Design A general challenge in the design of tangible interfaces is that their design requires a diverse skill set including electrical engineering, software development and interaction design. This required mix of skills, and particularly the electrical engineering component, often makes it difficult for researchers to build a tangible interface that actually works well enough to be used in a real setting like a classroom. Some common rules have been given ([8], [11]) but these rules depreciate the tangible character of the object, for example, by making it more abstract.

Efficiency We observed that the tangible interface was faster over the whole experiment. We believe that one of the explanation for this result is related to the use of graspable objects that increase the communication bandwidth with the computer. As shown in figure 9, users can benefit from this property to improve their ability to manipulate objects. However, as the number of manipulations increases, subjects are more likely to use special actions (like the lasso selection) that come with the touch interface, thus increasing their performance.

Familiarity It is clear that tangible interfaces take advantage of our familiarity with the everyday world. However, as touch interfaces gain in popularity, the gap between the two interfaces should be reduced.

Persistence One strength of the tangible objects is that they exist outside the space defined by the projection screen. This characteristic allows users to increase their work space. Conversely, the non-persistence of virtual objects allows them to be decoupled from the real world where physical laws apply. The interface designers then enjoy more 


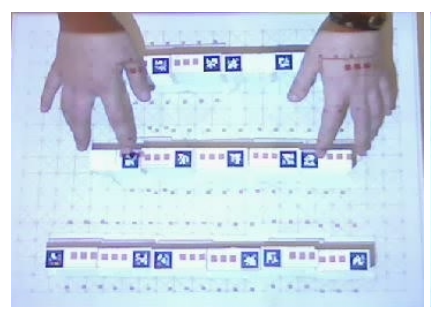

(a)

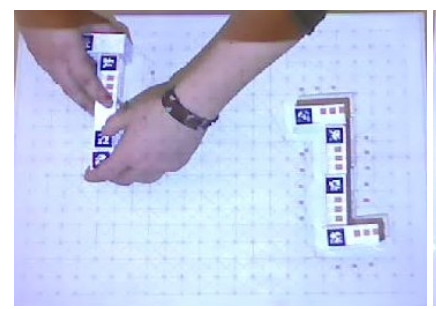

(c)

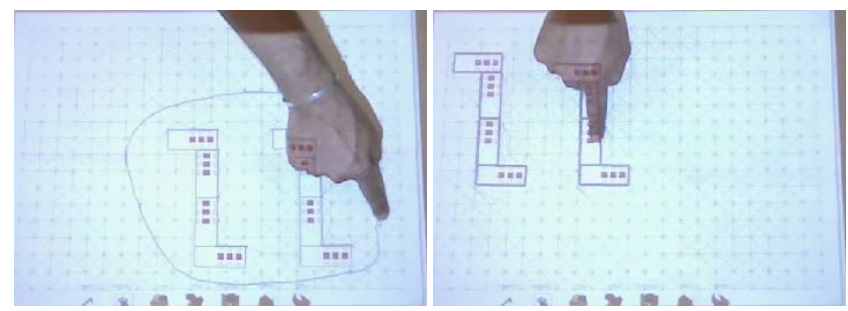

(e)

(f)

Figure 9. (a,b,c,d) Subjects use the graspable nature of the tangible object to manipulate them. (e,f) The same functionality can be transferred to the touch interface by implementing a special tool, like the lasso selection.

freedom and flexibility in implementing actions that can increase user performances if used adequately.

Feedback Different types of feedback were generated by the two interfaces. While the feedback produced by the touch interface was purely visual, the tangible interface also generates sounds and tactile feedback. These properties allow us to manipulate objects without having to look at them, which is probably one of the reasons why the tangible interface was the fastest one for our experiment. These tactile feedback techniques have been already studied in the literature [14] but we strongly believe that more work has to be done to see how to provide a more efficient feedback mechanism with touch interfaces.

We wish to emphasize that we have restricted our study to strictly quantifiable measurements (such as the time taken to complete an action). Previous studies ([18], [13]) have indeed incorporated less quantifiable measurements such as learning benefits, collaborative aspects and fun generated by the interface.

\section{CONCLUSION}

The findings presented in this paper showed that tangible interfaces are very effective at spatial layout tasks such as the one used for this experiment. We are not the first ones to show that tangible interfaces are particularly suitable for a specific domain. It has been already well accepted among researchers that tangible and touch interfaces have different affordances, which leaves us with an open question. How can we transfer the qualities of one technology to another ? Answering this question implies identifying and quantifying the costs and benefits of each technology in order to choose the most adequate for a chosen task, in the same way that biologists established a complete map of the heterochomatic genetic functions in Drosophila. Coupling touch and tangible technologies is another possible lead for researchers.

Further research should study how additional mechanisms can be implemented to transfer functionalities from one interface to another. The transfer of functionality between different interfaces is not guaranteed and must be empirically validated. For instance, typing performance suffers when tactile feedback from the keyboard is replaced by audio feedback. Likewise, our study showed that the Lasso selection could be substituted for the graspable nature of tangible objects in some situations.

Finally, our experiments showed that users might not be comfortable with fancy gestures. Indeed, many subjects did not use the Lasso selection even though the video tutorial explained how to use it. Interaction design is a general challenge for any interface but it seems that more work has to be done for touch interfaces, especially because new generation phones are making touch a fundamental way to communicate.

\section{ACKNOWLEDGEMENTS}

This project is part of Dual-T, a leading house on technologies for vocational training, funded by the Swiss Department of Economic Affairs. Special thanks to Pooya Pakzad for conducting the experiments, Olivier Guédat for his help for the practical tasks and Son Do Lenh for useful feedback in developing the software. We also would like to thank the following people for their involvement in the project : Quentin Bonnard, Cécile Bucher, David Bréchet, Bertrand Schneider, Florence Colomb, Marc-Antoine Nüssli.

\section{REFERENCES}

1. P.-A. Albinsson and S. Zhai. High precision touch screen interaction. In CHI '03: Proceedings of the SIGCHI conference on Human factors in computing systems, pages 105-112, New York, NY, USA, 2003. ACM.

2. J. Brewer, A. Williams, and P. Dourish. A handle on what's going on: combining tangible interfaces and ambient displays for collaborative groups. In TEI '07: Proceedings of the 1st international conference on Tangible and embedded interaction, pages 3-10, New York, NY, USA, 2007. ACM.

3. S. K. Card, W. K. English, and B. J. Burr. Evaluation of mouse, rate-controlled isometric joystick, step keys, and text keys, for text selection on a crt. 
Human-computer interaction: a multidisciplinary approach, pages 386-392, 1987.

4. M. Fiala. Artag revision 1. a fiducial marker system using digital techniques. Technical report, National Research Council of Canada, November 2004.

5. P. M. Fitts and J. R. Peterson. Information capacity of discrete motor responses. J Exp Psychol, 67:103-112, February 1964.

6. G. W. Fitzmaurice and W. Buxton. An empirical evaluation of graspable user interfaces: towards specialized, space-multiplexed input. In Proceedings of the SIGCHI conference on Human factors in computing systems, pages 43-50, New York, NY, USA, 1997. ACM.

7. G. W. Fitzmaurice, H. Ishii, and W. A. S. Buxton. Bricks: laying the foundations for graspable user interfaces. In CHI '95: Proceedings of the SIGCHI conference on Human factors in computing systems, pages 442-449, New York, NY, USA, 1995. ACM.

8. M. Fjeld. Graspable interfaces: Establishing design principles, phd thesis, 1998.

9. C. Forlines, D. Wigdor, C. Shen, and R. Balakrishnan. Direct-touch vs. mouse input for tabletop displays. In CHI '07: Proceedings of the SIGCHI conference on Human factors in computing systems, pages 647-656, New York, NY, USA, 2007. ACM.

10. J. Y. Han. Low-cost multi-touch sensing through frustrated total internal reflection. In UIST '05: Proceedings of the 18th annual ACM symposium on User interface software and technology, pages 115-118, New York, NY, USA, 2005. ACM.

11. H. Ishii. Tangible bits: beyond pixels. In Proceedings of the 2nd international conference on Tangible and embedded interaction, pages $\mathrm{xv}-\mathrm{xxv}$, New York, NY, USA, 2008. ACM.

12. S. Jordà, G. Geiger, M. Alonso, and M. Kaltenbrunner. The reactable: exploring the synergy between live music performance and tabletop tangible interfaces. In TEI '07: Proceedings of the 1st international conference on Tangible and embedded interaction, pages 139-146, New York, NY, USA, 2007. ACM.

13. P. Marshall. Do tangible interfaces enhance learning? In TEI '07: Proceedings of the 1st international conference on Tangible and embedded interaction, pages 163-170, New York, NY, USA, 2007. ACM.

14. A. Olwal, S. Feiner, and S. Heyman. Rubbing and tapping for precise and rapid selection on touch-screen displays. In CHI '08: Proceeding of the twenty-sixth annual SIGCHI conference on Human factors in computing systems, pages 295-304, New York, NY, USA, 2008. ACM.
15. A. J. Parkes, H. S. Raffle, and H. Ishii. Topobo in the wild: longitudinal evaluations of educators appropriating a tangible interface. In $\mathrm{CHI}$ '08: Proceeding of the twenty-sixth annual SIGCHI conference on Human factors in computing systems, pages 1129-1138, New York, NY, USA, 2008. ACM.

16. J. Patten and H. Ishii. Mechanical constraints as computational constraints in tabletop tangible interfaces. In CHI '07: Proceedings of the SIGCHI conference on Human factors in computing systems, pages 809-818, New York, NY, USA, 2007. ACM.

17. M. Rauterberg, M. Fjeld, H. Krueger, M. Bichsel, U. Leonhardt, and M. Meier. Build-it: a planning tool for construction and design. In $\mathrm{CHI}$ '98: $\mathrm{CHI} 98$ conference summary on Human factors in computing systems, pages 177-178, New York, NY, USA, 1998. ACM.

18. Y. Rogers, Y.-K. Lim, and W. R. Hazlewood. Extending tabletops to support flexible collaborative interactions. In TABLETOP '06: Proceedings of the First IEEE International Workshop on Horizontal Interactive Human-Computer Systems, pages 71-78, Washington, DC, USA, 2006. IEEE Computer Society.

19. A. Sears and B. Shneiderman. High precision touchscreens: Design strategies and comparisons with a mouse. International Journal of Man-Machine Studies, 34:593-613, 1991.

20. B. K. Slinker and S. A. Glantz. Multiple Linear Regression: Accounting for Multiple Simultaneous Determinants of a Continuous Dependent Variable. Circulation, 117(13):1732-1737, 2008.

21. C. Stauffer and W. Grimson. Adaptive background mixture models for real-time tracking. In Proceedings of the IEEE Computer Society Conference on Computer Vision and Pattern Recognition, volume 2, pages 246-252, 1999.

22. L. Terrenghi, D. Kirk, A. Sellen, and S. Izadi. Affordances for manipulation of physical versus digital media on interactive surfaces. In Proceedings of the SIGCHI conference on Human factors in computing systems, pages 1157-1166, New York, NY, USA, 2007. ACM.

23. J. Underkoffler and H. Ishii. Urp: a luminous-tangible workbench for urban planning and design. In $\mathrm{CHI}$ '99: Proceedings of the SIGCHI conference on Human factors in computing systems, pages 386-393, New York, NY, USA, 1999. ACM.

24. P. Wellner. Interacting with paper on the digitaldesk. Commun. ACM, 36(7):87-96, 1993.

25. D. Wigdor, C. Forlines, P. Baudisch, J. Barnwell, and C. Shen. Lucid touch: a see-through mobile device. In UIST '07: Proceedings of the 20th annual ACM symposium on User interface software and technology, pages 269-278, New York, NY, USA, 2007. ACM. 